\title{
Quadratic recursive convolution (QRC) in dispersive media simulation of finite-difference time-domain (FDTD)
}

\author{
Jinzu Ji ${ }^{1} \quad$ Hui Xue $^{2} \quad$ Tong Kin-Fai $^{3} \quad$ Peilin Huang ${ }^{* 1}$ \\ (1 School of Aeronautic Science and Engineering, Beihang University, Beijing, 100191, China) \\ (2 Beijing System Engineering Institute, Beijing, 100101, China) \\ (3 Department of Electronic and Electrical Engineering, University College London, London WC1E6BT, UK) \\ ( ${ }^{*}$ corresponding author, huang_pei_lin@126.com)
}

\begin{abstract}
This paper presents a novel formulation for dispersive media computation in finite-difference time-domain (FDTD). Motivated by conventional recursive convolution (RC) methods in handling convolution integral, the method name quadratic RC (QRC) makes improvement in the approximation of electric field in convolution integral. The electric field is approximated by quadratic function determined by the fields at three time steps at current, next and former. Via quadratic interpolation, the convolution integral result is approximated by the linear combination of three electric fields, rather than two fields in trapezoidal RC (TRC) or piecewise linear RC (PLRC) and one field in constant $\mathrm{RC}(\mathrm{CRC})$. Because three electric fields is required for the convolution integral, the method needs two more back level storage of the electric fields to fulfill the recursion process. Numerical demonstrations of Debye and Drude slab's transmission and reflection coefficients demonstrate the efficiency and accuracy of the novel method.

Key words: finite-difference time-domain (FDTD); quadratic recursive convolution (QRC); quadratic interpolation; recursive convolution $(\mathrm{RC})$

1 Introduction

Finite-difference time-domain (FDTD) has been widely used in electromagnetic computation since Yee presented his famous staggered grid and leapfrog updating iteration [1]. One of the most important advantages of the FDTD is that the broadband response can be obtained via only one computation. The FDTD's stability analysis [2], computation area's truncation [3] method are developed in the last decades and the application area is enhanced. To simplify the difference equations and make electric and magnetic fields more symmetry, equi-dimensional form is proposed [4]. The FDTD makes it possible that the analysis of interaction of electromagnetic waves with material bodies of arbitrary shape.
\end{abstract}

The classical FDTD assumed that the permittivity, permeability and conductivity are constant and independent of frequency. However, for many real materials of interest, these parameters vary significantly with frequency, such as snow, ice, soil, see water and plasma. Extension of FDTD to frequency-dependent materials has received relatively high attention since 1990s and many methods were raised to simulate dispersive media [5-11]. The methods to compute dispersive media generally can be summarized into three categories: recursive convolution (RC), auxiliary differential equation (ADE), and $\mathrm{Z}$ transform (ZT). RC expresses the constitutive relation in time domain with a convolution integral which is calculated by recursively $[5-7,10]$. ADE introduces auxiliary differential equation which is updated along with Maxwell's equation in time domain $[12,13]$. ZT transform the constitutive relation to Z domain and then transforms to time domain, in which shift operator is used to create difference equations [9].

There are some other aspects relative to the dispersive media's computation. Unconditionally stable complex envelope split-step wave-equation FDTD formulation was presented and it is based on incorporating the Strang time-splitting approach into the exponential evolution operator scheme [14]. This method can model dispersive electromagnetic application. A rational-fraction dispersion model for efficient simulation of dispersive material in FDTD was proposed [15]. The model can simulate optical properties of arbitrary linear dispersive media over a wide wavelength range. Weakly conditionally stable (WCS) was involved in ADE-FDTD to release the time step's limit and can be used in computing general hybrid dispersive media [16]. Berenger's perfectly matched layer (PML) was also extended to truncate dispersive media [3].

There are several RC formulation styles in computing dispersive media, including constant RC (CRC), trapezoidal RC (TRC) and piecewise linear RC (PLRC). The first proposed RC assumed that the electric field in the convolution integral is constant, so it is called CRC [5]. In TRC, the electric field in the convolution integral is approximated by the average of the field at the terminal of the interval [17]. PLRC assumes that the electric field is a linear function in the convolution interval [10]. Theoretical analysis and numerical demonstrations show that TRC and PLRC are more accurate than $\mathrm{CRC}$, but they need to store one more back level electric field in recursion process.

Motivated by the three RC styles, this paper presents a novel method named quadratic RC (QRC) in computation of dispersive media. In this paper, we shall show how to improve the electric field's approximation by binomial interpolation. In the $\mathrm{QRC}$, electric field in the convolution interval is approximated by quadratic function which is polynomial interpolation of three sampled electric fields which are current time step field, the field at next time step and former time step. The cost is that two more back level fields need to be stored to finish the recursion process. At last, numerical examples demonstrate the QRC's efficiency and accuracy.

Time factor $e^{j \omega t}$ is assumed and suppressed in this paper.

\section{Formulation of the convolution integral 2.1 General form of the RC approach}

The constitutive relation of electric flux density $\mathbf{D}$ and electric field intensity $\mathbf{E}$ is 


$$
\mathbf{D}(\omega)=\varepsilon_{0}\left(\varepsilon_{\infty}+\chi(\omega)\right) \mathbf{E}(\omega)
$$

where $\omega$ is angular frequency, $\varepsilon_{0}$ is permittivity of free space, $\varepsilon_{\infty}$ is infinite frequency relative permittivity, $\chi(\omega)$ is susceptibility which tends to zero when the frequency tends to infinitely large. To emphasize the frequency dependence of the fields, we keep argument $\omega$ with $\mathbf{D}$ and $\mathbf{E}$ in (1).

We can get the time domain constitutive relation by Fourier's transform which contains convolution integral, that is

$$
\mathbf{D}(t)=\varepsilon_{0}\left(\varepsilon_{\infty} \mathbf{E}(t)+\int_{0}^{t} \chi(t-\tau) \mathbf{E}(\tau) \mathrm{d} \tau\right)
$$

By using notation of $f^{n}=f(n \Delta t)$, where $f$ is an arbitrary field and $\Delta t$ is temporal increment, we get the discrete form of the constitutive relation at time step $n$

$$
\mathbf{D}^{n}=\varepsilon_{0}\left(\varepsilon_{\infty} \mathbf{E}^{n}+\int_{0}^{n \Delta t} \chi(n \Delta t-\tau) \mathbf{E}(\tau) \mathrm{d} \tau\right)
$$

The value of $\mathbf{D}$ at the next time step is

$$
\mathbf{D}^{n+1}=\varepsilon_{0}\left(\varepsilon_{\infty} \mathbf{E}^{n+1}+\int_{0}^{(n+1) \Delta t} \chi((n+1) \Delta t-\tau) \mathbf{E}(\tau) \mathrm{d} \tau\right)
$$

Difference of $\mathbf{D}^{n+1}$ and $\mathbf{D}^{n}$ is expressed as

$$
\begin{aligned}
& \mathbf{D}^{n+1}-\mathbf{D}^{n}=\varepsilon_{0} \varepsilon_{\infty}\left(\mathbf{E}^{n+1}-\mathbf{E}^{n}\right)+\varepsilon_{0} \int_{0}^{(n+1) \Delta t} \chi((n+1) \Delta t-\tau) \mathbf{E}(\tau) \mathrm{d} \tau-\varepsilon_{0} \int_{0}^{n \Delta t} \chi(n \Delta t-\tau) \mathbf{E}(\tau) \mathrm{d} \tau \\
& =\varepsilon_{0} \varepsilon_{\infty}\left(\mathbf{E}^{n+1}-\mathbf{E}^{n}\right)-\varepsilon_{0} \Psi^{n}+\varepsilon_{0} \mathbf{I}_{\chi}^{n}
\end{aligned}
$$

where

$$
\begin{gathered}
\mathbf{I}_{\chi}^{n}=\int_{n \Delta t}^{(n+1) \Delta t} \chi((n+1) \Delta t-\tau) \mathbf{E}(\tau) \mathrm{d} \tau \\
\Psi^{n}=\int_{0}^{n \Delta t}[\chi(n \Delta t-\tau)-\chi((n+1) \Delta t-\tau)] \mathbf{E}(\tau) \mathrm{d} \tau=\int_{0}^{n \Delta t} \Delta \chi(n \Delta t-\tau) \mathbf{E}(\tau) \mathrm{d} \tau
\end{gathered}
$$

where

$$
\Delta \chi(t)=\chi(t)-\chi(t+\Delta t)
$$

For dispersive media whose $\Delta \chi(t)$ is exponential function $\Delta \chi=e^{-\alpha t}$, we have $\Delta \chi(t+\Delta t)=\Delta \chi(t) e^{-\alpha \Delta t}$. Therefore, for this kind of dispersive media, $\Psi^{n}$ can be updated by recursion

where

$$
\begin{aligned}
& \Psi^{n+1}=\int_{0}^{(n+1) \Delta t} \Delta \chi((n+1) \Delta t-\tau) \mathbf{E}(\tau) \mathrm{d} \tau \\
& =\int_{0}^{n \Delta t} \Delta \chi((n+1) \Delta t-\tau) \mathbf{E}(\tau) \mathrm{d} \tau+\int_{n \Delta t}^{(n+1) \Delta t} \Delta \chi((n+1) \Delta t-\tau) \mathbf{E}(\tau) \mathrm{d} \tau \\
& =\int_{0}^{n \Delta t} e^{-\alpha \Delta t} \Delta \chi(n \Delta t-\tau) \mathbf{E}(\tau) \mathrm{d} \tau+\mathbf{I}_{\Delta \chi}^{n} \\
& =e^{-\alpha \Delta t} \Psi^{n}+\mathbf{I}_{\Delta \chi}^{n}
\end{aligned}
$$

$$
\mathbf{I}_{\Delta \chi}^{n}=\int_{n \Delta t}^{(n+1) \Delta t} \Delta \chi((n+1) \Delta t-\tau) \mathbf{E}(\tau) \mathrm{d} \tau
$$

Up till now, we did no approximation in the convolution integral. The key points of RC lie in the two integrals $\mathbf{I}_{\chi}^{n}$ and $\mathbf{I}_{\Delta x}^{n}$.

\subsection{The CRC, TRC and PLRC's approximations}

Because $\mathbf{E}(\tau)$ is sampled as the integer time step in the FDTD, we cannot know the exact value in the interval $[n \Delta t,(n+1) n \Delta t]$ and can only do some approximation. For the CRC method, $\mathbf{E}(\tau)$ is approximated by a constant $\mathbf{E}^{n+1}$ in the integral interval (6) and (10), so we get

where

$$
\begin{aligned}
\mathbf{I}_{\chi}^{n} & =\mathbf{E}^{n+1} \chi_{0} \\
\mathbf{I}_{\Delta \chi}^{n} & =\mathbf{E}^{n+1} \Delta \chi_{0}
\end{aligned}
$$

$$
\begin{gathered}
\chi_{0}=\int_{0}^{\Delta t} \chi(\tau) \mathrm{d} \tau \\
\Delta \chi_{0}=\int_{0}^{\Delta t} \Delta \chi(\tau) \mathrm{d} \tau
\end{gathered}
$$

In conduction of (13) and (14), variable substitution of $(n+1) \Delta t-\tau \rightarrow \tau$ is utilized.

For the TRC method, $\mathbf{E}(\tau)$ is approximated by $\left(\mathbf{E}^{n+1}+\mathbf{E}^{n}\right) / 2$ in the integral interval (6) and (10), so we get 


$$
\begin{aligned}
\mathbf{I}_{\chi}^{n} & =\frac{\mathbf{E}^{n+1}+\mathbf{E}^{n}}{2} \chi_{0} \\
\mathbf{I}_{\Delta \chi}^{n} & =\frac{\mathbf{E}^{n+1}+\mathbf{E}^{n}}{2} \Delta \chi_{0}
\end{aligned}
$$

For the PLRC method, $\mathbf{E}(\tau)$ is approximated by a piecewise linear function expressed by

$$
\mathbf{E}(\tau)=\mathbf{E}^{n+1} \frac{\tau-(n+1) \Delta t}{-\Delta t}+\mathbf{E}^{n} \frac{\tau-n \Delta t}{\Delta t}
$$

Substituting (17) into (6) and (10), we have

$$
\begin{gathered}
\mathbf{I}_{\chi}^{n}=\mathbf{E}^{n+1}\left(\chi_{0}-\xi_{0}\right)+\mathbf{E}^{n} \xi_{0} \\
\mathbf{I}_{\Delta \chi}^{n}=\mathbf{E}^{n+1}\left(\Delta \chi_{0}-\Delta \xi_{0}\right)+\mathbf{E}^{n} \Delta \xi_{0}
\end{gathered}
$$

where

$$
\begin{aligned}
\xi_{0} & =\int_{0}^{\Delta t} \chi(\tau) \frac{\tau}{\Delta t} \mathrm{~d} \tau \\
\Delta \xi_{0} & =\int_{0}^{\Delta t} \Delta \chi(\tau) \frac{\tau}{\Delta t} \mathrm{~d} \tau
\end{aligned}
$$

The above RC methods approximate $\mathbf{E}(\tau)$ by different manners. The CRC uses only one terminal's value of the integral interval and has the least accuracy. The TRC and PLRC use both the terminal values of the integral interval and are more accurate. The cost of TRC and PLRC is that one more back level of electric field should be stored in the memory to complete the recursion process. In PLRC, convolution $\xi_{0}$ and $\Delta \xi_{0}$ are also required.

\subsection{The QRC's approximation}

In the QRC method, $\mathbf{E}(\tau)$ in the integral interval $[n \Delta t,(n+1) \Delta t]$ is approximated by quadratic interpolation of $\mathbf{E}^{n+1}, \mathbf{E}^{n}, \mathbf{E}^{n-1}$, which is

$$
\mathbf{E}(\tau) \approx \mathbf{E}^{n+1} \frac{(\tau-(n-1) \Delta t)(\tau-n \Delta t)}{2 \Delta t^{2}}+\mathbf{E}^{n} \frac{(\tau-(n-1) \Delta t)(\tau-(n+1) \Delta t)}{-\Delta t^{2}}+\mathbf{E}^{n-1} \frac{(\tau-n \Delta t)(\tau-(n+1) \Delta t)}{2 \Delta t^{2}}
$$

By variable substitution of $s=(n+t) \Delta t-\tau$, (22) can be expressed as

$$
\begin{aligned}
& \mathbf{E}(\tau) \approx \frac{\mathbf{E}^{n+1}}{2 \Delta t^{2}}(2 \Delta t-s)(\Delta t-s)+\frac{\mathbf{E}^{n}}{-\Delta t^{2}}(2 \Delta t-s)(-s)+\frac{\mathbf{E}^{n-1}}{2 \Delta t^{2}}(\Delta t-s)(-s) \\
& =\frac{\mathbf{E}^{n+1}}{2 \Delta t^{2}}\left(s^{2}-3 \Delta t s+2 \Delta t^{2}\right)+\frac{\mathbf{E}^{n}}{-\Delta t^{2}}\left(s^{2}-2 \Delta t s\right)+\frac{\mathbf{E}^{n-1}}{2 \Delta t^{2}}\left(s^{2}-\Delta t s\right)
\end{aligned}
$$

By the same substitution, (6) can be expressed as

$$
\mathbf{I}_{\chi}^{n}=\int_{0}^{\Delta t} \chi(s) \mathbf{E}(\tau) \mathrm{d} s
$$

Substituting (23) into (24), considering definition of $\chi_{0} \quad \xi_{0}$ in (13) and (20), we get

where

$$
\mathbf{I}_{\chi}^{n}=\mathbf{E}^{n+1}\left(\chi_{0}-\frac{3}{2} \xi_{0}+\zeta_{0}\right)+\mathbf{E}^{n}\left(2 \xi_{0}-2 \zeta_{0}\right)+\mathbf{E}^{n-1}\left(-\frac{1}{2} \xi_{0}+\zeta_{0}\right)
$$

$$
\zeta_{0}=\frac{1}{2} \int_{0}^{\Delta t} \chi(\tau)\left(\frac{\tau}{\Delta t}\right)^{2} \mathrm{~d} \tau
$$

By the same procedure, $\mathbf{I}_{\Delta \chi}^{n}$ can be similarly constructed and we have

$$
\mathbf{I}_{\Delta \chi}^{n}=\mathbf{E}^{n+1}\left(\Delta \chi_{0}-\frac{3}{2} \Delta \xi_{0}+\Delta \zeta_{0}\right)+\mathbf{E}^{n}\left(2 \Delta \xi_{0}-2 \Delta \zeta_{0}\right)+\mathbf{E}^{n-1}\left(-\frac{1}{2} \Delta \xi_{0}+\Delta \zeta_{0}\right)
$$

where

$$
\Delta \zeta_{0}=\frac{1}{2} \int_{0}^{\Delta t} \Delta \chi(\tau)\left(\frac{\tau}{\Delta t}\right)^{2} \mathrm{~d} \tau
$$

In QRC, $\mathbf{I}_{\chi}^{n}$ and $\mathbf{I}_{\Delta \chi}^{n}$ is a linear combination of $\mathbf{E}^{n+1}, \mathbf{E}^{n}, \mathbf{E}^{n-1}$.

\subsection{Unified expressions of the RCs}

Actually, $\mathbf{I}_{\chi}$ and $\mathbf{I}_{\Delta \chi}$ of the RC, TRC, PLRC and QRC can be expressed by the unified forms, which are

$$
\begin{gathered}
\mathbf{I}_{\chi}^{n}=A_{\chi} \mathbf{E}^{n+1}+B_{\chi} \mathbf{E}^{n}+C_{\chi} \mathbf{E}^{n-1} \\
\mathbf{I}_{\Delta \chi}^{n}=A_{\Delta \chi} \mathbf{E}^{n+1}+B_{\Delta \chi} \mathbf{E}^{n}+C_{\Delta \chi} \mathbf{E}^{n-1}
\end{gathered}
$$

where $A_{\chi}, B_{\chi}, C_{\chi}, A_{\Delta \chi}, B_{\Delta \chi}$ and $C_{\Delta \chi}$ are coefficients, which are chosen properly in the different style of the 
RC methods. The coefficients are listed in the Table 1.

Table 1 Coefficients of the CRC, the TRC, the PLRC and the QRC

\begin{tabular}{|c|c|c|c|c|}
\hline & $\mathrm{CRC}$ & TRC & PLRC & QRC \\
\hline$A_{\chi}$ & $\chi_{0}$ & $\frac{\chi_{0}}{2}$ & $\chi_{0}-\xi_{0}$ & $\chi_{0}-\frac{3}{2} \xi_{0}+\zeta_{0}$ \\
\hline$B_{\chi}$ & 0 & $\frac{\chi_{0}}{2}$ & $\xi_{0}$ & $2 \xi_{0}-2 \zeta_{0}$ \\
\hline$C_{\chi}$ & 0 & 0 & 0 & $-\frac{1}{2} \xi_{0}+\zeta_{0}$ \\
\hline$A_{\Delta \chi}$ & $\Delta \chi_{0}$ & $\frac{\Delta \chi_{0}}{2}$ & $\Delta \chi_{0}-\Delta \xi_{0}$ & $\Delta \chi_{0}-\frac{3}{2} \Delta \xi_{0}+\Delta \zeta_{0}$ \\
\hline$B_{\Delta \chi}$ & 0 & $\frac{\Delta \chi_{0}}{2}$ & $\Delta \xi_{0}$ & $2 \Delta \xi_{0}-2 \Delta \zeta_{0}$ \\
\hline$C_{\Delta x}$ & 0 & 0 & 0 & $-\frac{1}{2} \Delta \xi_{0}+\Delta \zeta_{0}$ \\
\hline
\end{tabular}

From Table 1, we can see that the four styles of RC methods have the same stencil. The difference of the four RC methods is that the six coefficients' choices. For conventional RC methods including the CRC, TRC and PLRC, $C_{\chi}$ and $C_{\Delta \chi}$ are ignored and $\mathbf{E}(\tau)$ in the integral interval is approximated by a constant or a linear function. In QRC method, $\mathbf{E}(\tau)$ in the interval is approximated by a quadratic function and the approximation is more accurate.

\section{Fields updating}

By using Ampere's law $\nabla \times \mathbf{H}=\frac{\partial \mathbf{D}}{\partial t}$ and the approximation of $\left.\frac{\partial \mathbf{D}}{\partial t}\right|_{t=(n+1 / 2) \Delta t} \approx \frac{\mathbf{D}^{n+1}-\mathbf{D}^{n}}{\Delta t}$, we have

$$
\varepsilon_{0} \varepsilon_{\infty}\left(\mathbf{E}^{n+1}-\mathbf{E}^{n}\right)-\varepsilon_{0} \Psi^{n}+\varepsilon_{0}\left(A_{\chi} \mathbf{E}^{n+1}+B_{\chi} \mathbf{E}^{n}+C_{\chi} \mathbf{E}^{n-1}\right)=\Delta t \nabla \times \mathbf{H}^{n+1 / 2}
$$

By introducing (31), (5) and (29) is used. Then $\mathbf{E}^{n+1}$ can be expressed in the explicit form

$$
\mathbf{E}^{n+1}=\frac{\varepsilon_{\infty}-B_{\chi}}{\varepsilon_{\infty}+A_{\chi}} \mathbf{E}^{n}+\frac{-C_{\chi}}{\varepsilon_{\infty}+A_{\chi}} \mathbf{E}^{n-1}+\frac{1}{\varepsilon_{\infty}+A_{\chi}} \boldsymbol{\Psi}^{n}+\frac{\Delta t}{\varepsilon_{0}\left(\varepsilon_{\infty}+A_{\chi}\right)} \nabla \times \mathbf{H}^{n+1 / 2}
$$

where $\boldsymbol{\Psi}^{n}$ can be updated recursively by (9).

As we aforementioned, (9) is validated only when $\Delta \chi(t)$ of the media is an exponential function. We can demonstrate that for Debye and Drude media, $\Delta \chi(t)$ satisfy the requirement. Susceptibility of Debye media is

$$
\chi^{\text {Debye }}(\omega)=\frac{\Delta \varepsilon}{1+j \omega \tau_{0}}
$$

where $\Delta \varepsilon=\varepsilon_{s}-\varepsilon_{\infty}$ and $\varepsilon_{s}$ is static permittivity, $\tau_{0}$ is relaxation time. Susceptibilities of Drude is

$$
\chi^{\text {Drude }}(\omega)=\frac{\omega_{\mathrm{p}}^{2}}{j \omega(j \omega+v)}
$$

where $\omega_{\mathrm{p}}$ is radian plasma frequency, $v$ is collision frequency. The susceptibilities of time domain can be obtained via Fourier's transform

$$
\begin{gathered}
\chi^{\text {Debye }}(t)=\frac{\Delta \varepsilon}{\tau_{0}} e^{-t / \tau_{0}} U(t) \\
\chi^{\text {Drude }}(t)=\frac{\omega_{\mathrm{p}}^{2}}{v}\left(1-e^{-v t}\right) U(t)
\end{gathered}
$$

where $U(t)$ is Heaviside function which is 1 when $t \geq 0$ and 0 when $t<0$. From definition of $\Delta \chi$ in (8), We have

$$
\Delta \chi^{\text {Debye }}(t)=\frac{\Delta \varepsilon}{\tau_{0}} e^{-t / \tau_{0}}\left(1-e^{-t / \tau_{0}}\right) U(t)
$$




$$
\Delta \chi^{\text {Drude }}(t)=-\frac{\omega_{\mathrm{p}}^{2}}{v} e^{-v t}\left(1-e^{-v \Delta t}\right) U(t)
$$

They are both exponential function and thus (9) is valid for Debye and Drude media.

Integrals of $\chi_{0}, \xi_{0}, \zeta_{0}, \Delta \chi_{0}, \Delta \xi_{0}$ and $\Delta \zeta_{0}$ for Debye and Drude media are listed in Table 2.

Table 2 Integrals of $\chi_{0}, \xi_{0}, \zeta_{0}, \Delta \chi_{0}, \Delta \xi_{0}$ and $\Delta \zeta_{0}$

\begin{tabular}{|c|c|c|}
\hline & Debye & Drude \\
\hline$\chi_{0}$ & $\Delta \varepsilon\left(1-e^{-\frac{\Delta t}{\tau_{0}}}\right)$ & $\frac{\omega_{p}^{2}}{v} \Delta t-\frac{\omega_{p}^{2}}{v^{2}}\left(1-e^{-v \Delta t}\right)$ \\
\hline$\xi_{0}$ & $\frac{\tau_{0}}{\Delta t} \Delta \varepsilon\left(1-\left(1+\frac{\Delta t}{\tau_{0}}\right) e^{-\frac{\Delta t}{\tau_{0}}}\right)$ & $\frac{\omega_{p}^{2}}{v} \frac{\Delta t}{2}-\frac{\omega_{p}^{2}}{v^{3} \Delta t}\left(1-(1+v \Delta t) e^{-v \Delta t}\right)$ \\
\hline$\zeta_{0}$ & $\frac{\tau_{0}^{2}}{\Delta t^{2}} \Delta \varepsilon\left(1-\left(1+\frac{\Delta t}{\tau_{0}}+\frac{\Delta t^{2}}{2 \tau_{0}^{2}}\right) e^{-\frac{\Delta t}{\tau_{0}}}\right)$ & $\frac{\omega_{p}^{2}}{v} \frac{\Delta t}{6}-\frac{\omega_{p}^{2}}{v^{4} \Delta t^{2}}\left(1-\left(1+v \Delta t+\frac{v^{2} \Delta t^{2}}{2}\right) e^{-v \Delta t}\right)$ \\
\hline$\Delta \chi_{0}$ & $\Delta \varepsilon\left(1-e^{-\frac{\Delta t}{\tau_{0}}}\right)^{2}$ & $-\frac{\omega_{p}^{2}}{v^{2}}\left(1-e^{-v \Delta t}\right)^{2}$ \\
\hline$\Delta \xi_{0}$ & $\frac{\tau_{0}}{\Delta t} \Delta \varepsilon\left(1-\left(1+\frac{\Delta t}{\tau_{0}}\right) e^{-\frac{\Delta t}{\tau_{0}}}\right)\left(1-e^{-\frac{\Delta t}{\tau_{0}}}\right)$ & $-\frac{\omega_{p}^{2}}{v^{3} \Delta t}\left(1-(1+v \Delta t) e^{-v \Delta t}\right)\left(1-e^{-v \Delta t}\right)$ \\
\hline$\Delta \zeta_{0}$ & $\frac{\tau_{0}^{2}}{\Delta t^{2}} \Delta \varepsilon\left(1-\left(1+\frac{\Delta t}{\tau_{0}}+\frac{\Delta t^{2}}{2 \tau_{0}^{2}}\right) e^{-\frac{\Delta t}{\tau_{0}}}\right)\left(1-e^{-\frac{\Delta t}{\tau_{0}}}\right)$ & $-\frac{\omega_{p}^{2}}{v^{4} \Delta t^{2}}\left(1-\left(1+v \Delta t+\frac{v^{2} \Delta t^{2}}{2}\right) e^{-v \Delta t}\right)\left(1-e^{-v \Delta t}\right)$ \\
\hline
\end{tabular}

\section{Numerical validations}

\subsection{Plasma slab}

Numerical experiments of plasma and Debye media slabs' reflection and transmission coefficients are conducted to demonstrate the efficiency and accuracy of the QRC.

Plasma is a typical Drude media. The validation example used here is a classic example proposed in [6] and many authors used it to validate their algorithms. The slab is $15 \mathrm{~mm}$ thick. The plasma's frequency is $\omega_{p}=2 \pi \times 28.7 \times 10^{9} \mathrm{Rad} / \mathrm{s}$. The plasma's collision frequency is $v=20 \mathrm{GH} z$. The infinite permittivity is $\omega_{\infty}=1$. The simulation domain is 300 grids. The plasma occupies 50 250 grids of the grids. The grid's size is $\delta=75 \mu \mathrm{m}$. The time increment is $\Delta \mathrm{t}=0.125 \mathrm{ps}$, then we get the stable factor $s=\delta / c \Delta t=0.5$ of time difference, where $c$ is light speed in free space. The incident signal is differential Gaussian pulse, which is expressed as

$$
f(t)=\left(t-t_{0}\right) e^{-4 \pi\left(\left(t-t_{0}\right) / \tau\right)^{2}}
$$

where $\tau=60 \Delta t$ is shape factor and $t_{0}=3 \tau$.

The fields updating iteration is repeated 8000 times and the incident, reflected and transmitted waves at the bound of the slab were recorded. By Fourier transforms, we get response on frequency domain and then the complex reflection and transmission coefficients are obtained. The four styles of the RCs are all tested and compared. The results are shown in Figure 1 and Figure 2.

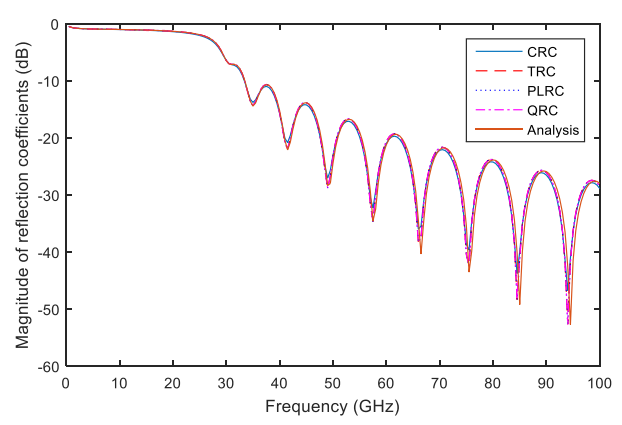

(a) Complex reflection coefficients' magnitude.

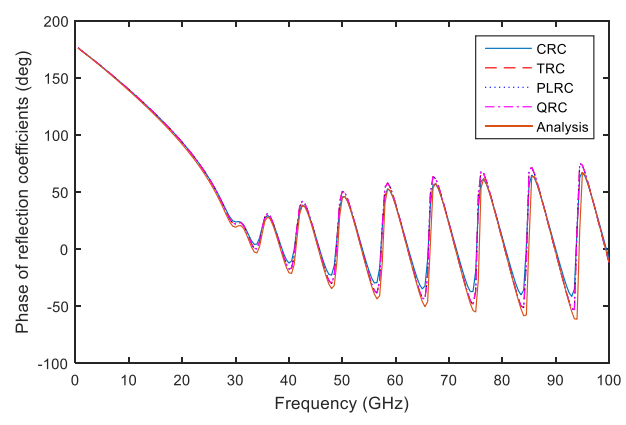

(b) Complex reflection coefficients' phase.

Figure 1 Complex reflection coefficients of Drude slab after 8000 time steps compared with the exact steady-state frequency domain solution. 


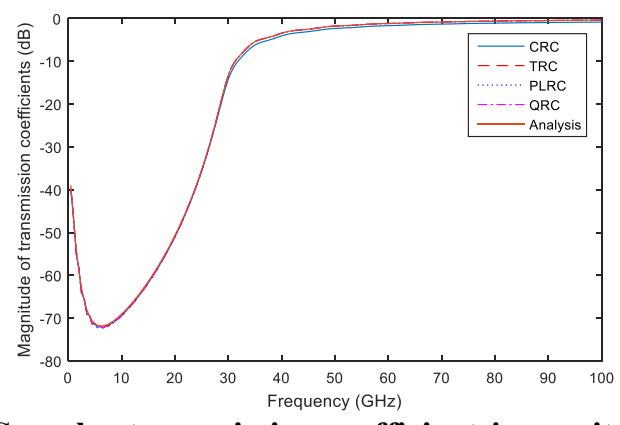

(a) Complex transmission coefficients' magnitude.

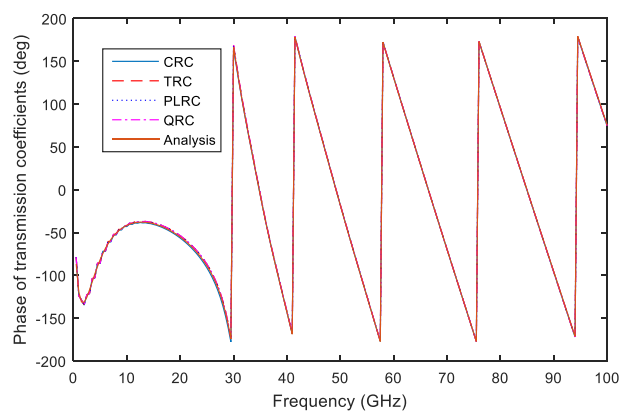

(b) Complex transmission coefficients' phase.

Figure 2 Complex transmission coefficients of Drude slab after 8000 time steps compared with the exact steady-state frequency domain solution.

From Figure 1 and Figure 2, we can see that QRC's results agree well with the exact frequency domain results. All of the RCs are comparatively accurate in Figure 1 and Figure 2. The detailed part between $80 \sim 85 \mathrm{GHz}$ of the Figure 1 (b) is given in Figure 3 to validate the accuracy more clearly.

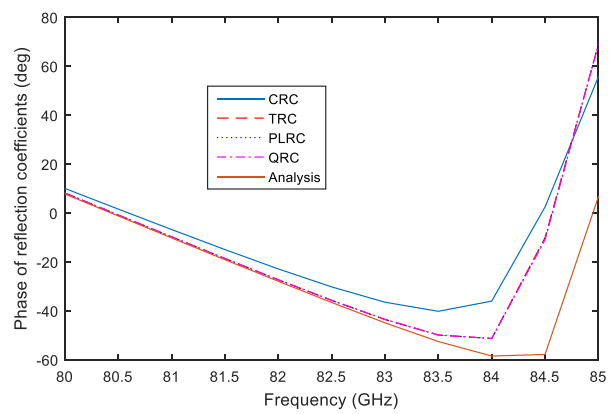

Figure 3 Detailed part of the reflection coefficients' phase of Drude slab.

From Figure 3, we can see that QRC is more accurate than CRC method. The QRC has almost the same accuracy with the TRC and the PLRC and the curves are almost superposed.

\subsection{Debye media slab}

Debye media slab's complex reflection and transmission coefficients are calculated by the QRC and other RCs. The grid settings and incident signal are the same with the former plasma slab experiment. The Debye media's parameters are $\tau_{0}=0.1 \mathrm{~ns}, \Delta \varepsilon=1$ and $\varepsilon_{\infty}=1$. The results are shown in Figure 4 and Figure 5 which show excellent agreement with analysis results.

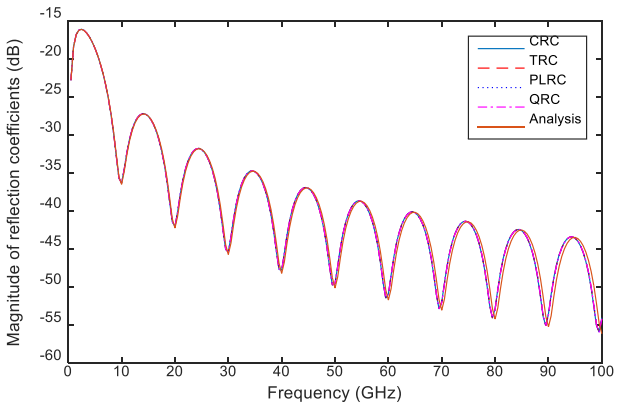

(a) Complex reflection coefficients' magnitude.

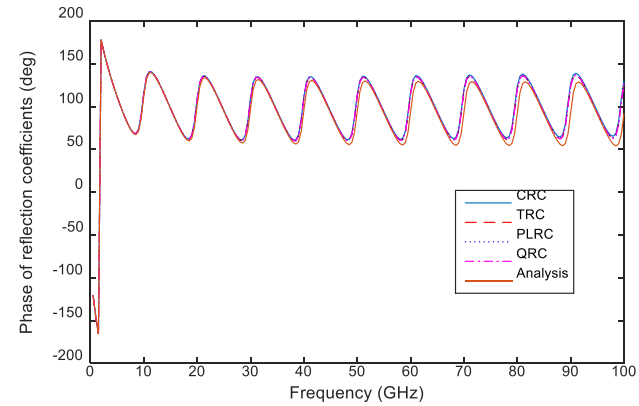

(b) Complex reflection coefficients' phase.

Figure 4 Complex reflection coefficients of Debye slab after 8000 time steps compared with the exact steady-state frequency domain solution. 


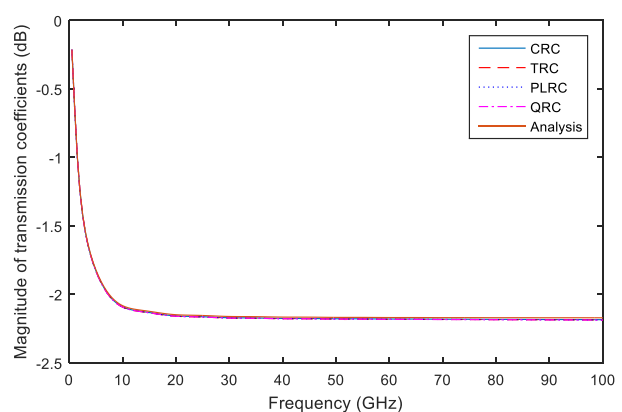

(a) Complex transmission coefficients' magnitude.

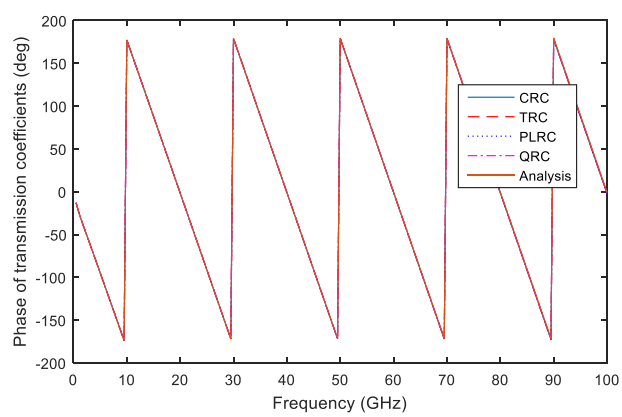

(b) Complex transmission coefficients' phase.

Figure 5 Complex transmission coefficients of Debye slab after 8000 time steps compared with the exact steady-state frequency domain solution.

\section{Conclusion}

This paper presents a novel recursive convolution method named QRC in computation of dispersive media using FDTD. The electric field in the convolution integral is approximated by a quadratic function determined by the adjacent three sampled fields. The integral result is a linearly combination of three electric fields at current, former and next integer time step. This method requires two more back level electric field, so need more memory in application. The numerical validations demonstrate the accuracy and efficiency of the QRC.

\section{Reference}

[1] K. S. Yee, "Numerical solution of initial boundary value problems involving maxwell's equations in isotropic media," Antennas and Propagation, IEEE Transactions on, vol. 14, pp. 302-307, 1966.

[2] Y. Baba and V. A. Rakov, "Applications of the FDTD Method to Lightning Electromagnetic Pulse and Surge Simulations," Ieee Transactions on Electromagnetic Compatibility, vol. 56, pp. 1506-1521, Dec 2014.

[3] F. L. Teixeira and W. C. Chew, "A general approach to extend Berenger's absorbing boundary condition to anisotropic and dispersive media," Antennas and Propagation, IEEE Transactions on, vol. 46, pp. 1386-1387, 1998.

[4] J. Ji, F. Liu, and P. Huang, "FINITE-DIFFERENCE TIME-DOMAIN OF EQUI-DIMENSIONAL FORM," Electronics World, vol. 120, pp. 24-27, May 2014.

[5] R. Luebbers, F. P. Hunsberger, K. S. Kunz, R. B. Standler, and M. Schneider, "A frequency-dependent finite-difference time-domain formulation for dispersive materials," Electromagnetic Compatibility, IEEE Transactions on, vol. 32, pp. 222-227, 1990.

[6] R. J. Luebbers, F. Hunsberger, and K. S. Kunz, "A frequency-dependent finite-difference time-domain formulation for transient propagation in plasma," Antennas and Propagation, IEEE Transactions on, vol. 39, pp. 29-34, 1991.

[7] R. J. Luebbers and F. Hunsberger, "FDTD for Nth-order dispersive media," Antennas and Propagation, IEEE Transactions on, vol. 40, pp. 1297-1301, 1992.

[8] O. P. Gandhi, B. Q. Gao, and J.-Y. Chen, "A frequency-dependent finite-difference time-domain formulation for general dispersive media," Microwave Theory and Techniques, IEEE Transactions on, vol. 41, pp. 658-665, 1993.

[9] R. Siushansian and J. LoVetri, "A comparison of numerical techniques for modeling electromagnetic dispersive media," Microwave and Guided Wave Letters, IEEE, vol. 5, pp. 426-428, 1995.

[10]D. F. Kelley and R. J. Luebbers, "Piecewise linear recursive convolution for dispersive media using FDTD," Antennas and Propagation, IEEE Transactions on, vol. 44, pp. 792-797, 1996.

[11]Q. Chen, M. Katsurai, and P. H. Aoyagi, "An FDTD formulation for dispersive media using a current density," Antennas and Propagation, IEEE Transactions on, vol. 46, pp. 1739-1746, 1998.

[12]J. L. Young and R. O. Nelson, "A summary and systematic analysis of FDTD algorithms for linearly dispersive media," Ieee Antennas and Propagation Magazine, vol. 43, pp. 61-77, Feb 2001.

[13]M. A. Alsunaidi and A. A. Al-Jabr, "A General ADE-FDTD Algorithm for the Simulation of Dispersive Structures," Ieee Photonics Technology Letters, vol. 21, pp. 817-819, May-Jun 2009.

[14]O. Ramadan, "Complex envelope split-step wave-equation FDTD formulations for dispersive electromagnetic applications," Electronics Letters, vol. 47, p. 250, 2011.

[15]L. Han, D. Zhou, K. Li, X. Li, and W. P. Huang, "A Rational-Fraction Dispersion Model for Efficient Simulation of Dispersive Material in FDTD Method," Journal of Lightwave Technology, vol. 30, pp. 2216-2225, Jul 2012.

[16]F. Liang, A. Chen, D. Zhao, and B.-Z. Wang, "ADE-WCS-FDTD METHOD FOR GENERAL DISPERSIVE MATERIALS AND PML IMPLEMENTATION," Microwave and Optical Technology Letters, vol. 56, pp. 2489-2495, Nov 2014.

[17]J. Shibayama, R. Ando, A. Nomura, J. Yamauchi, and H. Nakano, "Simple Trapezoidal Recursive Convolution Technique for the Frequency-Dependent FDTD Analysis of a Drude-Lorentz Model," Ieee 
Photonics Technology Letters, vol. 21, pp. 100-102, Jan-Feb 2009. 